\title{
PERSPECTIVES
}

VIEWPOINT

\section{Helicobacter pylori research: historical insights and future directions}

\author{
Kwong Ming Fock, David Y. Graham and Peter Malfertheiner
}

\begin{abstract}
Helicobacter pylori leads to chronic gastritis, peptic ulcer disease and gastric cancer. With increasing issues of antibiotic resistance and changing epidemiology of this pathogen, new approaches are needed for effective management. In 1984, Dr Barry Marshall and Dr Robin Warren reported the association of Helicobacter pylori with peptic ulcers in The Lancet-a discovery that earned them the Nobel prize in Physiology or Medicine in 2005_but what progress have we made since then? Here, we have invited three international experts to give their insights into the advances in $\mathrm{H}$. pylori research over the past 30 years and where research should be focused in the future.
\end{abstract}

Fock, K. M. et al. Nat. Rev. Gastroenterol. Hepatol. 10, 495-500 (2013); published online 11 June 2013; doi:10.1038/nrgastro.2013.96

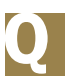

What do you think the most significant developments have been since the discovery of the association between $\mathrm{H}$. pylori and peptic ulcers 30 years ago?

Kwong Ming Fock. The recognition of $H$. pylori infection as the cause of gastric malignancies, in particular noncardia gastric adenocarcinoma and mucosalassociated lymphoid tissue lymphoma. ${ }^{1,2}$ An analysis of 12 prospective case-control studies concluded that the estimated relative risk of noncardia cancer associated with H. pylori infection was $5.9 .{ }^{3}$ On the basis of an average prevalence of $H$. pylori of $35 \%$ in developed countries and $85 \%$ in developing countries, it was estimated that $\sim 65-80 \%$ of noncardia gastric cancers were attributable to $H$. pylori infection and potentially preventable. ${ }^{3}$ The Asia-Pacific Gastric Cancer Consensus reviewed published data and performed a meta-analysis, which concluded that the pooled relative risk of developing gastric cancer after H. pylori

\section{Competing interests}

K. M. Fock declares no competing interests. D. Y. Graham declares associations with the following companies and organizations:

Baylor College of Medicine, Novartis, Otsuka Pharmaceuticals and RedHill Biopharma.

P. Malfertheiner declares associations with the following companies and organizations: AstraZeneca, Falk Foundation and Takeda. See the article online for details of these relationships. eradication was 0.56 (95\% CI $0.4-0.8){ }^{4}$ The Consensus recommended that populations in the Asia-Pacific region at high risk of gastric cancer (such as China, Japan and Korea) undergo population screening and treatment of $H$. pylori infection. The cut-off age for screening for $H$. pylori infection was recommended at 10-20 years before the initial incidence of gastric cancer began to rise rapidly in the local population. ${ }^{4}$ It must be recognized that prior to eradication, irreversible precancerous lesions might have already arisen and, in such cases, apart from $H$. pylori eradication, a strategy of continued surveillance is required. A paper from Taiwan in 2012 evaluated the benefit of mass eradication of $H$. pylori infection in reducing premalignant gastric lesions. ${ }^{5}$ This approach led to a marked reduction in the incidence of gastric atrophy resulting from chemoprevention (77.2\%; 95\% CI 72.3-81.2\%), whereas the reduction in intestinal metaplasia was not statistically significant. Mass eradicaton had an effectiveness of $25 \%$ during the chemoprevention period in reducing gastric cancer incidence when compared with the 5 -year period before chemoprevention and in the absence of endoscopic screening. ${ }^{5}$

David Y. Graham. The culture of $H$. pylori and the proof it was the primary cause of gastritis closed the loop on almost a century of research regarding gastritis and its associated diseases: gastric atrophy, peptic ulcer disease (PUD) and gastric cancer. ${ }^{6}$ Originally, the significance of the discovery was underestimated in part because the rich heritage of scholarship and research related to the associations between gastritis, acid secretion, gastric atrophy and gastric cancer remained largely invisible to the cadre of scientists who studied H. pylori. Manfred Comfort, who in 1951 summarized 50 years of research related to gastritis, acid secretion and gastric cancer, would likely have been amazed, or more accurately, appalled, to learn that the association of H. pylori with gastric cancer was considered to be important enough for publication in the New England Journal of Medicine as a new observation, rather than as a confirmation that elimination of gastric cancer was finally within our grasp. ${ }^{7}$ Even that observation might have been made years before if Grant Stemmermann had not repeatedly declined our invitation to test his serum samples because he believed that the association was unlikely. Conquering gastric cancer was further delayed because epidemiologists studying $H$. pylori remained blissfully unaware of the tremendous body of carefully done experiments linking gastritis and gastric cancer, and instead relied on the results of their serological assays to accurately assess risk. This belief resulted in greatly underestimating the attributable risk of $H$. pylori in gastric cancer; a belief that was only corrected in the past 10 years by the addition of CagA serology rather than discovery of the large body of prior knowledge based on histology. ${ }^{6}$ In 1938, Konjetzny wrote, "When we were able to prevent gastritis or treat it, we would be able to prevent ulcer and gastric cancer. Prophylaxis of gastritis means prophylaxis against ulcer and gastric cancer". 6 We again learn that what appears new to us, was often well understood by others.

In hindsight, the first 30 years of $H$. pylori have not been the glorious undertaking that could or should have rapidly confirmed and expanded the rich base of research done, previously resulting in a worldwide campaign to eradicate the infection and gastric cancer. Each step forward has been slow and hotly contested. ${ }^{8}$ For example, 


\begin{abstract}
The contributors*
Kwong Ming Fock is a Clinical Professor, Faculty of Medicine, National University of Singapore. He is Assistant Chief Executive Officer of Eastern Health Alliance and a Senior Consultant Gastroenterologist at Changi General Hospital (CGH). His previous positions at CGH include Head of Gastroenterology, Chief of Medicine, and Chairman of the Medical Board. Currently, Prof. Fock is also President of the Asia-Pacific Digestive Week Federation and member of World Gastroenterology Organisation Nominations Committee. Kwong Ming Fock has authored more than 150 papers and presented at numerous gastroenterology conferences and seminars. His research interests include $H$. pylori, GERD, gastric cancer and functional dyspepsia. He has authored the Asia-Pacific Consensus on $\mathrm{H}$. pylori infection, gastric cancer and GERD.

David Y. Graham is a Professor in the Departments of Medicine and Molecular Virology and Microbiology at the Michael E. DeBakey VA Medical Center and Baylor College of Medicine, Houston, TX, USA. He received his undergraduate degree from the University of Notre Dame in South Bend, IN, USA, his MD degree with honors from Baylor University College of Medicine. $\mathrm{He}$ is board certified in Medicine and Gastroenterology. He is a Past President of the American College of Gastroenterology and is the current Editor of the journal Helicobacter. His primary research interests are related to infections of the gastrointestinal tract including Helicobacter pylori, norovirus infections, and the possible infectious aetiology of IBD.

Peter Malfertheiner is Professor of Medicine and Chairman of the Department of Gastroenterology, Hepatology and Infectious Diseases at the Otto-von-Guericke University Magdeburg, Germany, an appointment he has held since 1995. He received his MD from the University of Bologna, Italy, in 1975, and gained subsequent medical experience at the Regional Hospital of Bozen, Italy, and the University Hospital of Ulm, Germany, before becoming a Research Fellow at the Mayo Clinic Gastrointestinal Unit in Rochester, MN, USA. Professor Malfertheiner was Chairman of the United European Gastroenterology (UEG) Federation in 2004, and in 2006 he was President of the UEG Week Congress and Chair of the Education Committee of the UEGF, and 2011 President of the German Society of Gastroenterology. His main research interests are Helicobacter pylori infection and related diseases, chronic inflammation and carcinogenesis, gastric cancer, hepatocellular cancer, GERD and chronic pancreatitis.

*The contributors are listed in alphabetical order.
\end{abstract}

H. pylori is probably the only important infectious disease in which when antibiotic resistance began to compromise treatment success, opinion leaders and 'consensus conferences' continued to recommend the increasingly ineffective regimens. ${ }^{9}$ Thus, whilst patients suffered unacceptably low treatment success, pharmaceutical profits and support for meeting and speakers programmes continued. In the future, I expect that social scientists will examine why the paradigm shift has taken so long and explore the role of vested interests, 'big PHARMA', the relation to changes in thinking and expiration of patents, and what must be called our collective poor scholarship that resulted in such an important disease to remain unconquered even after 30 years.

The significant developments in the past 30 years include: culture of $H$. pylori, development of noninvasive tests to identify active infection, development of effective antimicrobial therapies, confirmation of the role of $H$. pylori in gastritis-associated PUD and gastric cancer and proof that eradication of $H$. pylori would eliminate those diseases. In the USA, senator Edward Kennedy's personal involvement in pushing the FDA and the NIH to become involved in the $H$. pylori question is probably the most important and generally unrecognized aspect of this story. Finally, the most important recent event occurred in Spring 2013 in Japan where the presence of $H$. pylori infection is now accepted as an indication for treatment and country-wide eradication can begin. This eradication programme, the first anywhere, will ultimately also lead to the elimination of gastric cancer. I anticipate that other countries will ask that if Japan can rid itself of gastric cancer, why can't we?

Peter Malfertheiner. The discovery of Campylobacter pyloridis (renamed Helicobacter pylori in 1989 because of specific taxonomic characteristics) was the result of an attent and meticulous observation combined with the appropriate conclusion that this bacterial infection causes chronic active gastritis with the suggestion that it might be involved with PUD and gastric cancer. ${ }^{10,11}$

The discovery of $H$. pylori broke the dogma of the stomach as a sterile organ and was the starting point for re-examining gastric physiology and gastroduodenal pathologies. From the initial observations it required complex research efforts including basic and clinical research to demonstrate the role of $H$. pylori as a key trigger in PUD and the translation of this finding into clinical practice (reviewed elsewhere ${ }^{12}$ ). It has taken a long time for $H$. pylori to overcome many objections and become recognized as the principal causal factor in PUD, but more than 20 years later in 2005, the Nobel Prize was awarded to Dr Robin Warren and Dr Barry Marshall specifically for this discovery.

Bacterial virulence and host genetic factors as well as environmental conditions all contribute to linking the phenotypic expression of chronic gastritis with diverse clinical outcomes and have been unravelled over the past 30 years..$^{13} \mathrm{~A}$ clinical milestone was the causal association and cure of early stage gastric MALT lymphoma by $H$. pylori eradication, ${ }^{14,15}$ which has now been extended to also include advanced stages of this disease. ${ }^{16}$ Studies exploring the molecular mechanisms of chronic inflammation and immunity as well as cell signalling pathways for proliferation and apoptosis have contributed to our current understanding of the pathogenesis of gastric cancer. These findings will lead to improved diagnostic and therapeutic strategies to fight the dismal prognosis of this disease. ${ }^{17,18}$ So far, however, available strategies for the prevention of gastric cancer on a global scale have still not been implemented. ${ }^{19}$ Finally, complex treatment regimens have continuously been developed to meet the requirements of this difficult-to-treat chronic infection. ${ }^{20-22}$ Moreover, H. pylori infection has been found to have a role in extragastric diseases, opening a wide field for further exploration. ${ }^{23}$

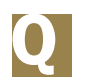

How do you think that the issue of antibiotic resistance should be tackled?

K.M.F. Understanding the local pattern of antibiotic resistance is important. In fact, each country should have up-to-date data on antibiotic susceptibility and the appropriate treatment regimens should be based on local antibiotic resistance patterns. The type of antibiotic resistance is a reflection of the wider use of antibiotics in the community. An example is metronidazole, which has a high resistance rate in most countries. In Japan, however, where its usage has been limited, the resistance rate is less than $5 \% .^{24}$ Although antibiotic susceptibility testing prior to treatment is an attractive concept, it is not practical in most clinical situations as clinicians utilize either the carbon urea breath test or rapid urease test to make the initial diagnosis. Antibiotic susceptibility testing must be 
considered in the context of treatment I agree with the approach advocated by the Maastricht IV consensus. ${ }^{22}$ In areas of high clarithromycin resistance (>15\%), bismuthcontaining quadruple therapies are recommended for first-line empirical treatment. If this regimen is not available, sequential treatment or a non-bismuth quadruple therapy is recommended. In regions with low clarithromycin resistance $(<15 \%)$, the standard 1-week triple therapy should still be prescribed..$^{22}$ In Singapore, where the clarithromycin resistance rate is low, a retrospective study showed that the success rate of triple therapy has remained at $\sim 90 \% .^{25}$ A prospective randomized controlled study comparing sequential and concomitant 10-day triple therapy revealed no differences between the therapies; these data were presented at DDW 2013.

\section{Each step forward has been slow and hotly contested... 77}

D.Y.G. Antibiotic resistance is neither a new problem nor one unique to $H$. pylori. Doctors in infectious diseases have dealt with this issue since the first antibiotics were introduced. The problem is largely a result of overuse of antibiotics generally, and to use of less than optimal regimens for $H$. pylori infections, specifically. As noted earlier, when other infections develop resistance, doctors change therapy. In H. pylori the response was to continue to recommend what was then known to be increasingly ineffective therapies. Journals even push investigators to compare new and effective regimens with known ineffective 'standard therapies' by demanding comparison trials and proof of better effectiveness rather than comparing to $100 \%$ treatment success. For example, in Italy, sequential therapy was repeatedly compared with triple therapy (known to be ineffective), followed by meta-analyses of those experiments done in the same population. At no time was it considered by clinicians testing sequential therapy that 10 -day sequential therapy might not be optimal in terms of dose of drug or duration of therapy. It took 10 years and investigators from Taiwan to recognize that 14-day sequential therapy was superior to 10-day therapy both overall and in the presence of metronidazole resistance. ${ }^{26}$ Possibly, gastroenterologists should relinquish development of new regimens to infectious disease specialists.
If one knows the pattern of resistance locally and the outcome of the available regimens in infections with susceptible and with resistant microorganisms, it is easy to choose a regimen that will reliably cure at least $90 \%$ of infections on the first shot. ${ }^{27,28}$ The answer to the question how should resistance be tackled is: logically and data based.

P.M. The most appropriate way would be the individualized approach by testing for $H$. pylori antibiotic resistance in the individual patient and selecting the appropriate therapy regimen. The most effective single antibiotic in current composite regimens is still clarithromycin, and a noninvasive test (that is faecal test with PCR for antibiotic resistance) would enable the appropriate selection of clarithromycin, depending on the test result. There is such a test available, but with obvious limitations in the practical execution for general use. ${ }^{29}$ The concept for such faecal antibiotic resistance tests is very appealing and might in the future be extended to other relevant antibiotics used for $H$. pylori treatment regimens, such as metronidazole and levofloxacin. Still remaining within the context of individual patient management, in those who require an upper gastrointestinal endoscopy during diagnostic workup, samples should be sent for microbiological analysis and resistance testing. A delay of 7-12 days before starting treatment remains appropriate as treatment can be tailored based on the resistance pattern and there is never urgency for treating chronic infection.

The current recommended and most practical strategy is the regional surveillance of antibiotic resistance for all antibiotics used in $H$. pylori eradication regimens. The selection of antimicrobials for first-line treatment can then be selected accordingly. Bismuth-based quadruple therapy is currently the most effective treatment in patients with clarithromycinresistant strains. Finally, it is critical that guidelines are not only set up but that they also get adequate dissemination and implementation in clinical practice for avoiding uncontrolled and improper use of antibiotic regimens for $H$. pylori eradication.

\section{ro Do you think that a vaccine for $\mathrm{H}$. pylori is feasible and the right approach?}

K.M.F. The concept of vaccination against a microbe to prevent disease occurrence is appealing. However, results thus far have been disappointing. At this point in time, I doubt that it will work. Given the fact that there is significant variation in the virulence potential and host response to infection, and the fact that clinical disease manifestation does not occur in most patients, a strategy of appropriate risk stratification must be explored. A well written review on this topic was recently published. ${ }^{30}$ The authors examined key factors as to why there is still no effective vaccines despite three decades of research: lack of enthusiasm among clinicians, research scientists, and public health authorities concerning the need for a vaccine; rudimentary understanding of the correlates of gastric immunity to $H$. pylori and of gastric mucosal immunology in general; the geographical heterogeneity of the H. pylori genome; and insufficient pharmaceutical industry support. ${ }^{30}$

D.Y.G. We lack reliable data regarding the rate of reinfection in developing countries where infection is almost universal and sanitation is lax; areas where we believe that reinfection would likely undermine antibiotic-based treatments and a vaccine would be the best option. We also need studies regarding the utility of community or regionwide eradication programmes. ${ }^{30}$ Although a preventive or preventivetherapeutic vaccine is desirable, considerable data suggest one is possible, and problems seem to be surmountable, there is essentially no funding available for vaccine development. The 'right approach' would probably be to put those interested persons together, identify the roadblocks (such as local downregulation of the immune response in the stomach) and fund targeted research.

\section{4 ...our collective poor} scholarship... resulted in such an important disease remain[ing] unconquered ... after 30 years... 77

P.M. H. pylori is special in establishing its chronic persistence in the human stomach by escaping specific and nonspecific immune responses of the host. Developing a vaccine is the best approach for: primary prevention of the infection and it might even find a way into therapy to enhance the immune response beyond the spontaneous and apparently insufficient immune mechanisms that occur during natural infection. Indeed, in animal models, 


\section{The discovery of H. pylori broke the dogma of the stomach as a sterile organ... 77}

H. pylori vaccines have proven to be effective in the prevention of the infection as well as in the treatment of an already established infection. ${ }^{31}$ Vaccine development in humans, however, faces severe challenges and has not yet been successful. Vaccines that have been tested in small numbers of infected or noninfected humans to this point have been predominantly based on the delivery of formulations with the bacterial colonization factor urease, or whole cell antigens with the essential conjunction of an adjuvant for 'empowering' the host immune response. ${ }^{32,33}$ The most common route for antigen delivery has been oral, although nasal, rectal and parenteral routes have also been tested. A humoral immune response and the induction of urease antibodies were elicited in several studies but in spite of this response, protection against H. pylori infection was not obtained. ${ }^{34,35}$ Several challenges in vaccine development need to be addressed and they include the selection of an adequate human model (that is, challenge studies in healthy volunteers versus field studies), the selection of H. pylori antigens in their optimal combinations, identification of adjuvant substances for enhancement of the immune response and the optimal route of vaccine administration. I believe there is a chance for a vaccine and we should go for it. Committed financial support for performing this research is unfortunately difficult to obtain.

\section{What effect do you think $\mathrm{H}$. pylori eradication will have on the remaining microbiota in the gut? Do you think that there are any potential clinical implications?}

K.M.F. I do not foresee any long-term effect on the remaining microbiota in the gut. Transient changes are inevitable during treatment but adverse events, such as pseudomembranous colitis, rarely occur as a result of antibiotic use. Indeed, it is quite clear that these changes are transient, and that generally microflora or gut flora revert back to normal within a month. ${ }^{36}$

D.Y.G. I do not think that this question is important or even very interesting. H. pylori causes a variety of different intragastric milieus ranging from highly acidic associated with duodenal ulcer to gastric atrophy where the stomach becomes populated with numerous different organisms and $H$. pylori is either absent or becomes a minor population. The stomach acts as a gatekeeper to prevent entry of microorganisms into the rest of the gastrointestinal tract and these different milieus can enhance, eliminate, or reduce its effectiveness even to the point where the stomach becomes a reservoir for bacterial contamination of the rest of the gastrointestinal tract.

Overall, the health of a human population without $H$. pylori is superior to one with $H$. pylori. The questions about effects of microbiota actually triggered the question regarding whether $H$. pylori infections might have positive benefits. These arguments boil down to the effect of $H$. pylori as a biological antisecretory agent or its having a role in regard to the hygiene hypothesis (that is $H$. pylori antigens being important for priming the immune system). H. pylori acquisition is associated with poor household hygiene such that it is either involved or a surrogate of poor household hygiene. Nothing kills a beautiful hypothesis quicker than a good experiment. The hypothesis regarding a possible role in the hygiene hypothesis has been answered in a 'natural experiment' in parts of Malaysia where hygiene is generally poor but $H$. pylori is virtually absent, making it possible to uncouple exposure to $H$. pylori antigens from the hygiene hypothesis. The dire events proposed by elimination of $H$. pylori infection, such as an increase in childhood asthma and atopy, are not present despite the absence of $H$. pylori-the hypothesis was tested and found wanting, consistent with $H$. pylori not being involved but rather is a surrogate for the hygiene hypothesis. ${ }^{37}$

Under certain conditions, $H$. pylori can act as a biological antisecretory agent. In those instances, the infection results in reduced acid secretion and even the development of atrophy and diseases related to an increased oesophageal acid load, such as symptomatic gastro-oesophageal reflux and adenocarcinoma of the oesophagus, become rare, as does duodenal ulcer. ${ }^{38}$ Unfortunately, there is a trade-off as atrophic gastritis is also the precursor lesion for gastric cancer. For example, in 1930 when H. pylori and atrophic gastritis were both common in the USA, gastric cancer was the most common cancer. By 2013, H. pylori, atrophic gastritis and gastric cancer are all rare, but adenocarcinoma of the oesophagus (a complication of gastro-oesophageal reflux) has increased. Although the increase is large as a fold increase, it was previously an extremely rare disease and oesophageal adenocarcinoma is now a rare disease concentrated in white men, especially older white man. Therefore, an extremely common cancer in men and women of all races (gastric cancer) was traded for a rare cancer in white men. Clearly, the rise in adenocarcinoma of the oesophagus is a new problem to be addressed but the answer is not to return to the era when gastric cancer is the most common cancer in our population. Incidentally, in Malaysia, oesophageal adenocarcinoma and $H$. pylori are both rare-again opposite to what had been predicted. In reality, none of the dire consequences of $H$. pylori eradication have actually occurred.

\section{4 ...treatment regimens should be based on local antibiotic resistance patterns 77}

P.M. This area is fascinating and one of current intensive research. One focus needs to be directed to the question of how $H$. pylori affects the rest of the gastric microbiome, and a second one of how $H$. pylori eradication influences gut microbiota in general. There are few reports on the composition of other gastric microbiota in the presence and absence of $H$. pylori in humans and animal models, ${ }^{39,40}$ but we know nothing about their functional role. The phenotype of $H$. pylori-induced chronic active gastritis, whether antrum predominant or in the form of atrophic pangastritis, differs in gastric acid production. The consequent changes in the luminal milieu will probably affect intestinal bacterial composition, intestinal bacterial overgrowth, clinical symptoms and even metabolic consequences.

A recent study has reported important changes in the microbiotic composition after gastric bypass bariatric surgery and gives some indication on how relevant the stomach is for the regulation of gut microbiota. ${ }^{41}$ Following H. pylori eradication, changes in the gut microbiota composition and resistance have not been extensively investigated. ${ }^{42}$ The available experience does not indicate that antibiotic resistance is a lasting effect. We denoted short-term, but not long-term, changes in faecal microbiota of selected species after H. pylori eradication. ${ }^{36}$ 
Q How do you think that $\mathrm{H}$. pylori should be managed in developing countries with high prevalence, such as India?

K.M.F. There should not be any difference when $H$ pylori infection is diagnosed in the context of a clinical disease. It should be eradicated, with the treatment regimen based on local patterns of antibiotic resistance. As countries become more developed, with improvements in sanitation, transmission rates will decrease and the overall prevalence of infection will decrease, mirroring what has occurred in developed countries. The main issue here is the prevention of disease, such as gastric cancer. This approach must take into account the epidemiology of the disease in these countries. The Asia-Pacific Gastric Cancer Consensus recommended that, in populations at high risk of gastric cancer in the Asia-Pacific region, population screening and treatment of $H$. pylori infection was the strategy of choice. This approach will not be relevant in developing countries with low gastric cancer incidence rates, despite high $H$. pylori seroprevalence. This point was specifically highlighted in our Asia-Pacific guidelines. ${ }^{4}$

D.Y.G. First, we need to understand the problem including studies of transmission of the infection and of reinfection rates after eradication of $H$. pylori in individuals, households and groups such as villages. ${ }^{43}$ Ideally, these countries are the candidates for an effective preventive or even better a preventive and therapeutic vaccine. These countries also serve as the reservoir for $H$. pylori as immigrants from these countries bring their $H$. pylori infection with them. We might soon need to check immigrants for $H$. pylori and eradicate the infection when they move to developed countries.

\section{In reality, none of the}

\section{dire consequences of $H$. pylori eradication have actually occurred 97}

P.M. In spite of the fact that developing countries have the highest prevalence of $H$. pylori, they are facing other tremendous challenges from acute infections with a much higher priority and demand for medical interventions. Infectious diseases carrying a high mortality rate starting from childhood take priority in these countries. Complications related to $H$. pylori infection occur typically in adulthood and advanced age and, except for gastric cancer, they can be managed in a selective way.

$H$. pylori eradication is effective in curing PUD and a variety of other gastrointestinal pathologies, including dyspeptic symptoms and, therefore, in a country such as India with low gastric cancer incidence in most regions and limited healthcare resources, H. pylori treatment should be reserved for patients who become symptomatic. The strategy would be totally different in a developing country with high incidence of gastric cancer. In such circumstances the way forward is to implement screen and treat strategies, such as those recommended in the recent Asian-Pacific H. pylori consensus conference. ${ }^{44}$

'Q]

What do you think the next 10 years of research will bring?

K.M.F. I believe we will move from regimens that employ two antibiotics (currently called high therapy) to regimens containing three antibiotics selected on the basis of their bactericidal efficacy. Research might result in greater clarity about the choice of antibiotic regimens, and provide potentially less complicated regimens with shorter duration, which can improve patient compliance without affecting efficacy.

D.Y.G. Widespread H. pylori eradication programmes and elimination of gastric cancer from high prevalence areas. Hopefully, an effective vaccine for use in developing countries will be generated.

P.M. From a pragmatic point of view, it is essential to win the battle against gastric cancer. Basic mechanisms in H. pyloritriggered gastric carcinogenesis will be further elucidated by continuously evolving molecular technologies (such as microRNAs), which will eventually lead to new targets for diagnostic and therapeutic interventions. Further characterization of $H$. pylori virulence and host risk factors will enable clinicians to better define and tailor strategies for gastric cancer prevention in populations and individuals.

The role of $H$. pylori in directing the host immune response will have to be further addressed and better understood. An eye has to be kept on the further evolution of $H$. pylori infection within the changing environment and in its interactions with the intestinal microbiome and metabolome. The role of H. pylori in its contribution to food intake, metabolism as well as for potential benefits in atopic diseases cannot be ignored. New and selective drug development targeting specific and vulnerable sites of $H$. pylori are expected. The recent paper on the proton-gated urea channel of $H$. pylori paves the way in such directions. ${ }^{45}$ Lastly, I continue to hope in the successful development of a vaccine and for a future H. pylori-free healthy stomach for all.

Changi General Hospital, 2 Semei Street 3 , Singapore 529889 (K. M. Fock). Michael E. DeBakey Veterans Affairs Medical Center, 2002 Holcombe Boulevard, Houston,

TX 77030, USA (D. Y. Graham). Otto-vonGuericke-Universtität, Leipziger Strasse 44, Magdeburg 39120, Germany (P. Malfertheiner).

Correspondence to:

kwong_ming_fock@cgh.com.sg,

dgraham@bcm.edu,

peter.malfertheiner@med.ovgu.de

1. [No authors listed] Schistosomes, liver flukes and Helicobacter pylori. IARC Working Group on the evaluation of carcinogenic risks to humans. Lyon, 7-14 June 1994. IARC Monogr. Eval. Carcinog. Risks Hum. 61, 1-241 (1994).

2. Parkin, D. M. The global health burden of infection-associated cancers in the year 2002. Int. J. Cancer 118, 3030-3044 (2006).

3. Helicobacter and Cancer Collaborative Group. Gastric cancer and Helicobacter pylori: a combined analysis of 12 case control studies nested within prospective cohorts. Gut 49 , 347-353 (2001).

4. Fock, K. M. et al. Asia-Pacific consensus guidelines on gastric cancer prevention. J. Gastroenterol. Hepatol. 23, 351-365 (2008).

5. Lee, Y. C. et al. The benefit of mass eradication of Helicobacter pylori infection: a communitybased study of gastric cancer prevention. Gut 62, 676-682 (2013).

6. Graham, D. Y. \& Asaka, M. Eradication of gastric cancer and more efficient gastric cancer surveillance in Japan: two peas in a pod. J. Gastroenterol. 45, 1-8 (2010).

7. Comfort, M. W. Gastric acidity before and after development of gastric cancer: its etiologic, diagnostic and prognostic significance. Ann. Intern. Med. 36, 1331-1348 (1951).

8. Cotton, P. NIH consensus panel urges antimicrobials for ulcer patients, skeptics concur with caveats. JAMA 271, 808-809 (1994).

9. Graham, D. Y., Lu, H. \& Yamaoka, Y. Y. Therapy for Helicobacter pylori infection can be improved: sequential therapy and beyond. Drugs 68, 725-736 (2008).

10. Warren, J. R. \& Marshall, B. Unidentified curved bacilli on gastric epithelium in active chronic gastritis. Lancet 321, 1273-1275 (1983).

11. Marshall, B. J. \& Warren, J. R. Unidentified curved bacilli in the stomach of patients with gastritis and peptic ulceration. Lancet 1, 1311-1315 (1984).

12. Malfertheiner, P., Chan, F. K. \& McColl, K. E. Peptic ulcer disease. Lancet 374, 1449-1461 (2009).

13. Amieva, M. R. \& El-Omar, E. M. Hostbacterial interactions in Helicobacter pylori infection. Gastroenterology 134, 306-323 (2008). 
14. Wotherspoon, A. C., Ortiz-Hidalgo, C., Falzon, M. R. \& Isaacson, P. G. Helicobacter pylori-associated gastritis and primary B-cell gastric lymphoma. Lancet 338, 1175-1176 (1991).

15. Ruskoné-Fourmestraux, A. et al. Gastric extranodal marginal zone B-cell lymphoma of MALT. Gut 60 , 747-758 (2011).

16. Nakamura, S. et al. Long-term clinical outcome of gastric MALT Iymphoma after eradication of Helicobacter pylori: a multicentre cohort follow-up study of 420 patients in Japan. Gut 61, 507-513 (2012).

17. Hatakeyama, M. Helicobacter pylori and gastric carcinogenesis. J. Gastroenterol. 44, 239-248 (2009).

18. Correia, M., Machado, J. C. \& Ristimäki, A Basic aspects of gastric cancer. Helicobacter 14 (Suppl. 1), 36-40 (2009).

19. Mizuno, S. et al. Prescreening of a high-risk group for gastric cancer by serologically determined Helicobacter pylori infection and atrophic gastritis. Dig. Dis. Sci. 55, 3132-3137 (2010).

20. Vakil, N. \& Megraud, F. Eradication therapy for Helicobacter pylori. Gastroenterology 133, 985-1001 (2007).

21. Tepes, B., O'Connor, A., Gisbert, J. P. \& O'Morain, C. Treatment of Helicobacter pylori infection. Helicobacter 17 (Suppl. 1), 36-42 (2012).

22. Malfertheiner, P. et al. Management of Helicobacter pylori infection-the Maastricht IV/Florence Consensus Report. Gut 61 , 646-664 (2012).

23. Blaser, M. J. Equilibria of humans and our indigenous microbiota affecting asthma. Proc. Am. Thorac. Soc. 9, 69-71 (2012).

24. Kobayashi, I. et al. Changing antimicrobial susceptibility epidemiology of Helicobacter pylori strains in Japan between 2002 and 2005. J. Clin. Microbiol. 45, 4006-4010 (2007).

25. Ang, T. L. et al. Is there still a role for empiric first-line triple therapy using proton pump inhibitor, amoxicillin and clarithromycin for Helicobacter pylori infection in Singapore? Results of a time trend analysis. J. Dig. Dis. 14, 100-104 (2013).

26. Liou, J. M. et al. Sequential versus triple therapy for the first-line treatment of Helicobacter pylori: a multicentre, open-label, randomised trial. Lancet 381, 205-213 (2013).

27. Graham, D. Y. \& Rimbara, E. Understanding and appreciating sequential therapy for Helicobacter pylori eradication. J. Clin. Gastroenterol. 45, 309-313 (2011)

28. Rimbara, E., Fischbach, L. A. \& Graham, D. Y. Optimal therapy for Helicobacter pylori infections. Nat. Rev. Gastroenterol. Hepatol. 8, 79 (2011).

29. Vécsei, A. et al. Stool polymerase chain reaction for Helicobacter pylori detection and clarithromycin susceptibility testing in children. Clin. Gastroenterol. Hepatol. 8, 309-312 (2010).

30. Zhang, S., Moise, L. \& Moss, S. F. H. pylori vaccines: why we still don't have any. Hum. Vaccin. 7, 1153-1157 (2011).

31. Moss, S. F. et al. HelicoVax: epitope-based therapeutic Helicobacter pylori vaccination in a mouse model. Vaccine 29, 2085-2091 (2011).

32. Kabir, S. The current status of Helicobacter pylori vaccines: a review. Helicobacter 12 , 89-102 (2007).

33. Del Giudice, G., Malfertheiner, P. \& Rappuoli, R Development of vaccines against Helicobacter pylori. Expert Rev. Vaccines 8, 1037-1049 (2009).

34. Aebischer, T. et al. Correlation of T cell response and bacterial clearance in human volunteers challenged with Helicobacter pylori revealed by randomised controlled vaccination with Ty21a-based Salmonella vaccines. Gut 57, 1065-1072 (2008).

35. Malfertheiner, P. et al. Safety and immunogenicity of an intramuscular Helicobacter pylori vaccine in noninfected volunteers: a phase I study. Gastroenterology 135, 787-795 (2008).

36. Bühling, A., Radun, D., Muller, W. A. \& Malfertheiner, P. Influence of antiHelicobacter triple therapy with metronidazole, omeprazole and clarithromycin on intestinal microflora. Aliment. Pharmacol. Ther. 15 , 1445-1452 (2001).

37. Lee, Y. Y., Mahendra Raj, S. \& Graham, D. Y. Helicobacter pylori infection-a boon or a bane: lessons from studies in a low-prevalence population. Helicobacter http:///dx.doi.org/ 10.1111/hel.12058.
38. Liu, Y., Akiyama, J. \& Graham, D. Y. Current understandings of Helicobacter pylori, peptic ulcer and gastroesophageal reflux disease. Minerva Gastroenterol. Dietol. 52, 235-248 (2006).

39. Bik, E. M. et al. Molecular analysis of the bacterial microbiota in the human stomach. Proc. Natl Acad. Sci. USA 103, 732-737 (2006).

40. Osaki, T. et al. Comparative analysis of gastric bacterial microbiota in Mongolian gerbils after long-term infection with $\mathrm{H}$. pylori. Microb. Pathog. 53, 12-18 (2012).

41. Graessler, J. et al. Metagenomic sequencing of the human gut microbiome before and after bariatric surgery in obese patients with type 2 diabetes: correlation with inflammatory and metabolic parameters. J. Pharmacogenomics http://dx.doi.org/10.1038/tpj.2012.43.

42. Jakobsson, $\mathrm{H}$. et al. Macrolide resistance in the normal microbiota after Helicobacter pylori treatment. Scand. J. Infect. Dis. 39, 757-763 (2007).

43. Thirumurthi, S. \& Graham, D. Y. Helicobacter pylori infection in India from a western perspective. Indian J. Med. Res. 136, 549-562 (2012).

44. Fock, K. M. et al. Second Asia-Pacific Consensus Guidelines for Helicobacter pylori infection. J. Gastroenterol. Hepatol. 24, 1587-1600 (2009)

45. Strugatsky, D. et al. Structure of the protongated urea channel from the gastric pathogen Helicobacter pylori. Nature 493, 255-258 (2013).

\section{Acknowledgements}

K. M. Fock has received grants from Health Sector Development Programme and National Medical Research Council. The author would also like to acknowledge assistance from Dr T. L. Ang in the preparation of this manuscript. D. Y. Graham is supported in part by the Office of Research and Development Medical Research Service Department of Veterans Affairs, Public Health Service grant DK56338 (which funds the Texas Medical Center Digestive Diseases Center), DK067366 and CA116845. The contents are solely the responsibility of the authors and do not necessarily represent the official views of the Veterans Affairs or $\mathrm{NIH}$. 